\title{
Association of preoperative levels of selected blood inflammatory markers with prognosis in gliomas
}

\author{
Raushan Auezova' \\ Nurzhan Ryskeldiev' \\ Aidos Doskaliyev' \\ Yerbol Kuanyshev' \\ Berik Zhetpisbaev' \\ Nurgul Aldiyarova' \\ Natalia Ivanova ${ }^{2}$ \\ Serik Akshulakov' \\ Lizette Auezova ${ }^{3}$ \\ 'Department of Pathology of the \\ Central Nervous System, National \\ Centre for Neurosurgery, Astana, \\ Kazakhstan; ${ }^{2}$ Polenov Russian \\ Scientific Research Institute of \\ Neurosurgery (a branch of Federal \\ Almazov North-West Medical \\ Research Centre), Ministry of \\ Health of the Russian Federation, \\ Saint-Petersburg, Russia; ${ }^{3}$ Bioactive \\ Molecules Research Group, \\ Department of Chemistry and \\ Biochemistry, Faculty of Sciences-II, \\ Lebanese University, Beirut, Lebanon
}

Correspondence: Lizette Auezova Bioactive Molecules Research Group, Department of Chemistry and Biochemistry, Faculty of Sciences-II, Lebanese University, Fanar, Beirut, Lebanon

Tel +96I 70172208

Email auezova_|@hotmail.com
This article was published in the following Dove Press journal:

OncoTargets and Therapy

II October 2016

Number of times this article has been viewed

Background: Red cell distribution width (RDW), neutrophil-lymphocyte ratio (NLR), and platelet count (PLT) routinely tested as part of the complete blood count are indicative of systemic inflammation. The prognostic significance of NLR and PLT in cancer was demonstrated in many studies while the role of RDW has been hardly investigated. The present study aimed to assess the association of RDW, NLR, and PLT with survival and tumor grade in glioma patients.

Methods: Clinical data from 178 patients with primary gliomas treated in a single institution were retrospectively analyzed. Receiver operating characteristic curves for cutoff value determination, Kaplan-Meier survival analysis, various bivariate tests, and univariate and multivariate Cox regression analyses were performed.

Results: Patients with high RDW ( $\geq 13.95)$ and NLR $(\geq 4)$ levels had worse overall survival (OS) (Wilcoxon test, $P<0.026$ and $P<0.003$, respectively) while the effect of thrombocytosis $\left(\geq 400 \times 10^{9} / \mathrm{L}\right)$ on prognosis was not significant. Besides, a strong association between RDW and NLR was found (Spearman's rho $=0.230, P<0.02 ; \chi^{2}=8.887, P<0.03$; Mann-Whitney $U$-test, $P<0.017)$. Moreover, RDW and NLR were significantly associated with tumor grade. In univariate Cox analysis, elevated NLR (hazard ratio, HR 1.385; confidence interval, CI 1.020-1.881, $P<0.037$ ), older age (HR 0.452, CI 0.329-0.621, $P<0$ ), and higher tumor grade (HR 1.624, CI 1.187-2.223, $P<0.002)$ were associated with poor outcomes. In the multivariate analysis, tumor grade, age, and Karnofsky performance score were identified as being independently prognostic for OS.

Conclusion: Preoperative NLR and RDW values can help to evaluate disease progression and outcomes in patients with gliomas, thereby contributing to patient follow-up optimization.

Keywords: glioma, neutrophil-lymphocyte ratio, platelet, red cell distribution width, survival, tumor grade

\section{Introduction}

It is well established that chronic inflammation is associated with increased susceptibility for cancer development and progression., ${ }^{1,2}$ Numerous inflammatory markers were proposed for outcome prediction including some blood clinical parameters such as neutrophil-lymphocyte ratio (NLR), platelet count (PLT), and red cell distribution width (RDW). These parameters routinely measured by automated hematology analyzer are always available and inexpensive; this is a major advantage regarding their application.

The prognostic role of NLR, the most studied parameter, was shown in multiple malignancies; a meta-analysis of 100 studies comprising 40,559 patients with different 
solid tumors has been recently published. ${ }^{3}$ The authors concluded that an optimal NLR cutoff value for outcome prediction is 4.0 .

The association of high PLT with reduced survival in cancer patients has also been investigated; however, there were contradictory findings regarding the cutoff point. ${ }^{4-6}$

RDW is a quantitative measure of anisocytosis, the variability in size of the circulating erythrocytes. It has been typically used in diagnosing certain anemias, in particular those that are associated with iron, vitamin B12, or folate deficiencies. A number of studies demonstrated the prognostic significance of RDW in patients with various cardiovascular pathologies and other chronic inflammatory conditions. ${ }^{7-9}$ Despite the findings that chronic inflammation is one of the underlying mechanisms of tumorigenesis, very few studies investigated RDW as a potential marker for predicting survival in cancer patients. ${ }^{10,11}$

Gliomas are primary central nervous system tumors accounting for $\sim 80 \%$ of all diagnosed tumors of the brain originating from brain parenchyma, with malignant gliomas constituting the most common brain tumors in adults. Despite advances in multimodal treatments, malignant gliomas are characterized by a short survival time.

No studies have been performed on the impact of RDW on outcomes in glioma patients. As for NLR and PLT, two studies were published on the prognostic significance of these parameters in patients with glioblastoma, the most aggressive primary brain tumor. ${ }^{12,13}$

The present study is the first to assess the association of preoperative RDW with outcomes and tumor grade in glioma patients. Also, for the first time, the prognostic significance of NLR and PLT was investigated in a cohort of patients who were presented with all histological types of glial tumors. Finally, the prognostic impact of these blood parameters together with other potential clinicopathological factors was investigated.

\section{Patients and methods Study population}

A retrospective analysis of a database of glioma patients who were surgically treated at the Department of Pathology of the CNS of National Center for Neurosurgery (Astana, Kazakhstan) from January 2009 to December 2012 was carried out. A total of 178 patients were enrolled in this study on the basis of the following criteria: histopathologically confirmed diagnosis of neuroepithelial tumor (according to WHO 2007 classification), preoperative full blood count (FBC) and vital status data available, normal hemoglobin level (120-180 g/L), absence of active infection, hematological, and autoimmune disorders, as well as absence of recent steroid treatment. Medical records were also reviewed for age at diagnosis, sex, tumor grade, side, volume, location (eloquent, near-eloquent, or non-eloquent), and Karnofsky performance score (KPS). Overall survival (OS) was defined as the time interval from histologic diagnosis to death, or last follow-up (September 30, 2015) for surviving patients.

Preoperative blood samples were routinely collected for FBC within 1-2 days before surgery. FBC including neutrophil count, lymphocyte count, PLT, and RDW was measured using an automated hematology analyzer (Abbott CD-1800; Abbott Laboratories, Abbott Park, IL, USA). NLR was calculated as neutrophil count divided by lymphocyte count using standard units. Cutoff values used for NLR, PLT, and RDW were $4.0,400 \times 10^{9} / \mathrm{L}$, and 13.95 , respectively.

Patients with low-grade gliomas (LGGs) were generally treated only surgically, and some patients had indications for radiotherapy. In patients with high-grade gliomas (HGGs), surgery was often followed by radiotherapy and/or chemotherapy; some HGG individuals were treated by surgery alone, because of the severity of their condition. In addition, anticonvulsants and steroids were used for symptomatic treatment.

All patients gave written informed consent to the study protocol, which was approved by the Ethics Commission of National Center for Neurosurgery (IORG0008395).

\section{Statistical analysis}

Continuous variables are presented as mean \pm standard error, and categorical variables are presented as frequencies and percentages. The relationships between RDW, NLR, and PLT were analyzed using bivariate tests including analyses of variance (ANOVAs), chi-square test for categorical variables, Spearman's rho for continuous variables, and MannWhitney $U$-test for continuous and categorical variables when considered together. Receiver operating characteristic (ROC) curves for OS prediction were plotted to determine the optimum cutoff points for RDW, NLR, PLT, and age. The area under curve (AUC) was used as an estimation of diagnostic accuracy. Kaplan-Meier OS curves were plotted for patients with high and low values of RDW, NLR, or PLT, and survival differences between the groups were analyzed by the generalized Wilcoxon test. Influence of RDW, NLR, PLT, and various clinicopathological variables on outcomes was assessed using univariate and multivariate Cox regression analyses. Statistical analyses were performed using the SPSS statistical software package, version 20.0 
(IBM Corporation, Armonk, NY, USA). For all the analyses, the significance level was set at $P<0.05$.

\section{Results}

\section{Patient characteristics}

A total of 178 patients, 93 (52.2\%) men and 85 (47.8\%) women, were included in the study. Their characteristics are presented in Table 1.

The mean age at diagnosis was $41.58 \pm 1.04$ years (range, 18-72 years). A ROC curve for OS prediction was plotted (Figure 1A) to determine the optimum cutoff value for age. This value was found to be 45 years, and the number of patients aged $\geq 45$ years was $68(38.2 \%)$.

Seventy-seven cases were diagnosed as LGGs including grade I (GI) and grade II (GII) tumors. The GI group was presented by dysembryoplastic neuroepithelial tumor (2), gangliocytoma (3), ganglioglioma (4), pleomorphic

Table I Patient characteristics

\begin{tabular}{|c|c|c|c|}
\hline Parameters & Mean (range) & Median & $\begin{array}{l}\text { Patient } \\
\text { number (\%) }\end{array}$ \\
\hline Age (years) & $41.58 \pm 1.04(18-72)$ & 41 & \\
\hline$<45$ & & & $110(61.8)$ \\
\hline$\geq 45$ & & & $68(38.2)$ \\
\hline \multicolumn{4}{|l|}{ Sex } \\
\hline Female & & & $85(47.8)$ \\
\hline Male & & & $93(52.2)$ \\
\hline \multicolumn{4}{|l|}{ Histology } \\
\hline LGG & & & $77(43.3)$ \\
\hline HGG & & & $101(56.7)$ \\
\hline KPS & $64.55 \pm 0.70(50-80)$ & 60 & \\
\hline$<70$ & & & $90(50.6)$ \\
\hline$\geq 70$ & & & $88(49.4)$ \\
\hline \multicolumn{4}{|l|}{ Tumor side } \\
\hline Left & & & $73(4 I .0)$ \\
\hline Right & & & $84(47.2)$ \\
\hline Middle & & & $15(8.4)$ \\
\hline Other & & & $6(3.4)$ \\
\hline \multicolumn{4}{|l|}{ Functional involvement } \\
\hline Eloquent & & & $110(61.8)$ \\
\hline Near-eloquent & & & $57(32.0)$ \\
\hline Non-eloquent & & & II (6.2) \\
\hline RDW & $14.09 \pm 0.15(10-27.40)$ & 13.55 & \\
\hline$<13.95$ & & & III (62.4) \\
\hline$\geq 13.95$ & & & $67(37.6)$ \\
\hline NLR & $4.66 \pm 0.25(0.67-19.14)$ & 3.75 & \\
\hline$<4$ & & & $92(51.7)$ \\
\hline$\geq 4$ & & & $86(48.3)$ \\
\hline Platelet count $\left(\times 10^{9} / \mathrm{L}\right)$ & $261.38 \pm 5.83(|| 6-5 \mid 8)$ & 252.00 & \\
\hline$<400$ & & & $166(93.3)$ \\
\hline$\geq 400$ & & & $12(6.7)$ \\
\hline
\end{tabular}

Abbreviations: LGG, low-grade gliomas; HGG, high-grade gliomas; KPS, Karnofsky performance score; RDW, red cell distribution width; NLR, neutrophillymphocyte ratio. xanthoastrocytoma (2), pilocytic astrocytoma (7), subependymal giant cell astrocytoma (2), and by subependymoma (1). The distribution of GII tumors was as follows: fibrillary astrocytoma (35), protoplasmic astrocytoma (8), oligodendroglioma (4), oligoastrocytoma (2), gemistocytic astrocytoma (3), mast cell astrocytoma (2), central neurocytoma (1), and ependymoma (1). HGGs (grades III and IV) were more abundant. Grade III (GIII) tumors were distributed as follows: anaplastic oligodendroglioma (8), anaplastic oligoastrocytoma (4), anaplastic astrocytoma (46), anaplastic ependymoma (7), anaplastic ganglioglioma (6), and choroid plexus carcinoma (2). Finally, the grade IV (GIV) group included 17 cases of glioblastoma, 3 neuroblastomas, 2 gliosarcomas, and 6 cases of medulloblastoma.

Eighty-eight patients had KPS $\geq 70$ at diagnosis. The majority of tumors were right/left-sided and located in eloquent or near-eloquent zones. Median tumor volume in the known 101 cases was $63 \mathrm{~cm}^{3}$ (mean $76.11 \pm 5.79 \mathrm{~cm}^{3}$, range $1-343 \mathrm{~cm}^{3}$ ).

The mean preoperative RDW was $14.09 \pm 0.15$. A ROC curve was constructed (Figure 1B) to optimize the cutoff point for RDW; it was determined to be 13.95. The mean NLR was $4.66 \pm 0.25$, and the mean PLT was $261.38 \pm 5.83 \times 10^{9} / \mathrm{L}$. This study failed to obtain the optimal cutoff values for NLR and PLT (NLR: AUC 0.523, 95\% CI 0.326-0.721, $P<0.797$; PLT: AUC 0.519, 95\% CI 0.350-0.688, $P<0.835)$. Therefore, the NLR cutoff value was chosen based on literature analysis; the most appropriate was 4.0.,14 For PLT cutoff, $400 \times 10^{9} / \mathrm{L}$ was used; this level, characteristic of thrombocytosis, was shown to be associated with poor prognosis in cancer patients. ${ }^{4,13}$

\section{Prognostic role of RDW, NLR, and PLT on Kaplan-Meier analysis}

The survival curves stratified by high levels of RDW or NLR are shown in Figure 2. Sixty-seven persons (37.6\%) had RDW $\geq 13.95$, which was associated with worse median OS of 20 months compared to 28 months for patients with RDW $<13.95(P<0.026)$ (Figure 2A). Importantly, no significant difference in OS was found between the RDW groups when the recommended cutoff value of 14.5 was used $(P<0.052)$.

NLR $\geq 4.0$ was observed in 86 patients (48.3\%) who were classified as high NLR individuals. Figure 2B shows the survival curves for patients with normal and high NLR values (median OS of 28 vs 17 months, respectively; $P<0.003$ ).

As for PLT, 12 (6.7\%) patients had preoperative thrombocytosis $\left(\geq 400 \times 10^{9} / \mathrm{L}\right)$ in this study. The differences in 

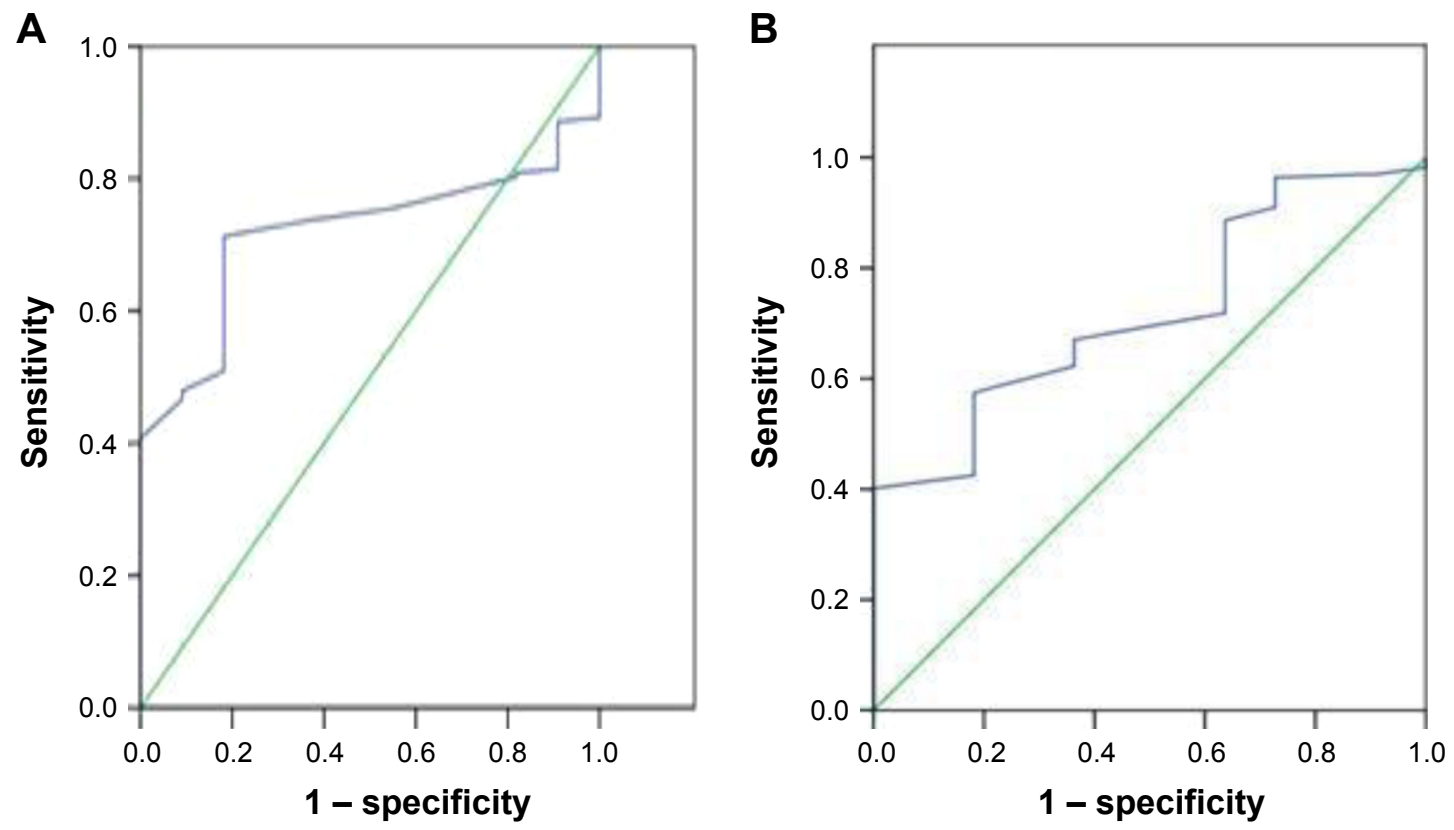

Figure I ROC curves for (A) age (AUC 0.7I9, 95\% Cl 0.624-0.8I4, P<0.0I5) and (B) RDW (AUC 0.720, 95\% Cl 0.592-0.847, P<0.0I5). Abbreviations: ROC, receiver operating characteristic; $\mathrm{AUC}$, area under curve; $\mathrm{Cl}$, confidence interval; RDW, red cell distribution width.

OS between the two groups stratified by $400 \times 10^{9} / \mathrm{L}$ were not significant $(P<0.480)$.

\section{Associations between RDW, NLR, and PLT}

Different statistical methods were used to study the relationships between RDW, NLR, and PLT. Associations between these parameters analyzed as continuous variables were assessed using Spearman analysis. A significant correlation was found between RDW and NLR (Spearman's rho $=0.230, P<0.02)$ while no significant correlation was obtained between NLR and PLT (Spearman's rho $=0.131$, $P<0.08)$.

Next, the studied hematological parameters were analyzed as categorical variables, and Pearson's chi-square test was applied to evaluate their potential associations. Notably, a significant association was found between RDW and NLR only when the RDW cutoff was adjusted from 14.5 to 13.95 $\left(\chi^{2}=8.887, P<0.03\right)$. A strong association was also obtained between RDW and PLT $\left(\chi^{2}=4.619, P<0.032\right)$. In addition,
A

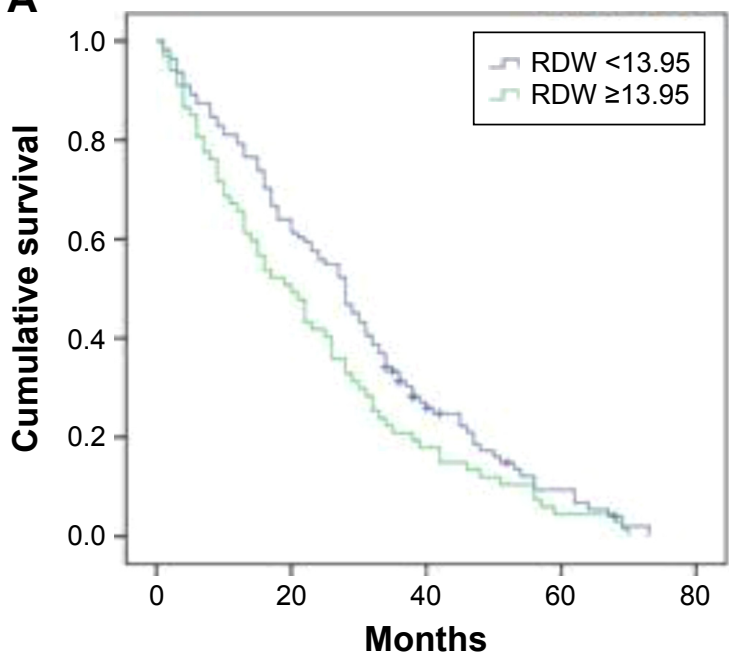

B

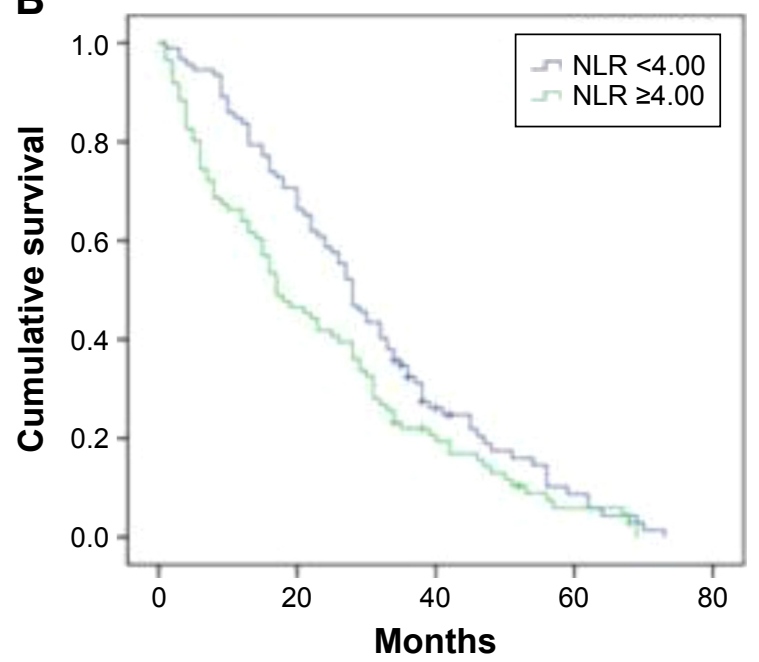

Figure 2 Overall survival Kaplan-Meier curves stratified by (A) RDW or (B) NLR. Abbreviations: RDW, red cell distribution width; NLR, neutrophil-lymphocyte ratio. 
a close association was observed between NLR and PLT $\left(\chi^{2}=3.669, P<0.055\right)$.

Moreover, significant differences between the groups were revealed by the Mann-Whitney $U$-test when the independent (grouping) variable was NLR (NLR vs RDW, $P<0.001$; NLR vs PLT, $P<0.030$ ). On the other hand, when PLT was selected as grouping variable, it was strongly associated only with RDW $(P<0.011)$. Importantly, with RDW as grouping variable, a significant association was determined only when the RDW groups were stratified by 13.95 (and not by 14.5); in this case, the level of RDW significantly correlated with NLR $(P<0.017)$. Thus, all the applied analyses demonstrated a strong association of RDW and NLR.

\section{Associations of RDW, NLR, and PLT with tumor grade}

Furthermore, the NLR, RDW, and PLT levels were compared among different tumor grades, using ANOVA with Duncan's post hoc test. Overall, NLR was shown to significantly increase in patients with GIV compared to GI, GII, or GIII $(P<0.043)$. On the other hand, when the studied parameters were analyzed as categorical ( $\chi^{2}$ test), a significant association was found between RDW and tumor grade $(P<0.033$ when graded as I-IV and $P<0.011$ when graded as LGG and $\mathrm{HGG})$.

Table 2 Univariate Cox regression analysis of factors affecting overall survival

\begin{tabular}{llll}
\hline $\begin{array}{l}\text { Variables in the } \\
\text { equation }\end{array}$ & P-value & $\begin{array}{l}\text { Hazard } \\
\text { ratio }\end{array}$ & $\begin{array}{l}95 \% \text { confidence } \\
\text { interval }\end{array}$ \\
\hline $\begin{array}{l}\text { Age } \\
\quad<45 \text { vs } \geq 45 \text { years }\end{array}$ & 0 & 0.452 & $0.329-0.621$ \\
$\begin{array}{l}\text { Sex } \\
\quad \text { Female vs male }\end{array}$ & 0.422 & 1.135 & $0.834-1.544$ \\
$\begin{array}{l}\text { Histology } \\
\quad \text { LGG vs HGG }\end{array}$ & 0.002 & 1.624 & $1.187-2.223$ \\
$\begin{array}{l}\text { KPS } \\
\quad<70 \text { vs } \geq 70\end{array}$ & 0.057 & 1.350 & $0.991-1.839$ \\
$\begin{array}{l}\text { Tumor side } \\
\text { Left vs right vs middle } \\
\text { vs other }\end{array}$ & 0.474 & & \\
$\begin{array}{l}\text { Functional involvement } \\
\text { Eloquent vs near-eloquent } \\
\text { vs non-eloquent }\end{array}$ & 0.373 & & \\
$\begin{array}{l}\text { RDW } \\
\quad<13.95 \text { vs } \geq 13.95\end{array}$ & 0.074 & 1.328 & $0.973-1.813$ \\
$\begin{array}{l}\text { NLR } \\
\quad<4 \text { vs } \geq 4\end{array}$ & & & \\
$\begin{array}{l}\text { Platelet count }\left(\times 10^{9} / L\right) \\
\quad<400 \text { vs } \geq 400\end{array}$ & 0.037 & 1.385 & $1.020-1.88 \mathrm{I}$ \\
\hline
\end{tabular}

Abbreviations: LGG, low-grade gliomas; HGG, high-grade gliomas; KPS, Karnofsky performance score; RDW, red cell distribution width; NLR, neutrophillymphocyte ratio.
Table 3 Multivariate Cox regression analysis of factors affecting overall survival

\begin{tabular}{llll}
\hline $\begin{array}{l}\text { Variables in the } \\
\text { equation }\end{array}$ & P-value & $\begin{array}{l}\text { Hazard } \\
\text { ratio }\end{array}$ & $\begin{array}{l}\text { 95\% confidence } \\
\text { interval }\end{array}$ \\
\hline $\begin{array}{l}\text { Age } \\
\quad<45 \text { vs } \geq 45 \text { years } \\
\begin{array}{l}\text { Histology } \\
\quad \text { LGG vs HGG }\end{array}\end{array}$ & 0 & 0.423 & $0.272-0.658$ \\
$\begin{array}{l}\text { KPS } \\
\quad<70 \text { vs } \geq 70\end{array}$ & 0.033 & 0.625 & $0.406-0.962$ \\
\hline
\end{tabular}

Abbreviations: LGG, low-grade gliomas; HGG, high-grade gliomas; KPS, Karnofsky performance score.

\section{Impact of NLR, RDW, PLT, and other clinicopathological variables on outcomes}

Factors affecting survival were studied using the Cox proportional hazards models. In univariate analysis, age $\geq 45$ years (hazard ratio, HR 0.452; 95\% confidence interval, 95\% CI 0.329-0.621; $P<0$ ), higher tumor grade (HR 1.624, 95\% CI 1.187-2.223, $P<0.002$ ), and NLR $\geq 4$ (HR 1.385, $95 \%$ CI $1.020-1.881, P<0.037)$ were found to be risk factors of poor outcomes (Table 2). Also, a close association with prognosis was demonstrated for KPS (HR 1.350, 95\% CI $0.991-1.839, P<0.057)$. In addition, RDW $\geq 13.95$ showed a tendency of poor prognosis $(P<0.074)$. Furthermore, by multivariate analysis, it was found that age (HR $0.423,95 \%$ CI $0.272-0.658, P<0$ ), tumor grade (HR 0.625 , 95\% CI 0.406-0.962, $P<0.033$ ), and KPS (HR 1.906, 95\% CI 1.244-2.921, $P<0.003)$ were independent prognostic factors (Table 3 ).

\section{Discussion}

The prognostic relevance of NLR in cancers has been demonstrated in many studies ${ }^{3,14}$ including a recent study on glioblastoma. ${ }^{12}$ The tumor microenvironment is characterized by a state of chronic inflammation, where tumor cells secrete diverse inflammatory mediators, thus accelerating tumor progression and metastasis. During this process, a close and complex interaction between cancer cells and stroma, including various immune cells and other stromal cells (eg, fibroblasts, mesenchymal stem cells, endothelial cells), takes place (reviewed in Hasselbalch ${ }^{2}$ and Sionov et a ${ }^{15}$ ). Neutrophils constitute a significant fraction of immune cells involved in the inflammatory process. In the context of cancer, neutrophils were shown to exert both pro- and antitumor activities suggesting a dual mode of activation. The factors that drive the balance between pro- and antitumorigenic neutrophils remain unknown. 
Very little is known about the prognostic significance of RDW in cancer. ${ }^{10,11} \mathrm{RDW}$ measures the variability in size of circulating erythrocytes; higher RDW values indicate greater variation in size. RDW elevation is markedly associated with increase of other inflammation markers, such as C-reactive protein, interleukin-6, and tumor necrosis factor- $\alpha .{ }^{16,17}$ The mechanisms underlying RDW fluctuations are poorly understood; oxidative stress and inflammation have been suggested as key factors. ${ }^{18}$

PLT is another convenient parameter that may help to predict patient survival. The primary function of platelets is to repair injured tissue through the recruitment of inflammatory cells. However, in chronic inflammation conditions, proinflammatory mediators such as cytokines increase platelet formation. Platelet activation stimulates tumor growth, dissemination, and angiogenesis. The interplay between platelets and tumor cells is complex and bidirectional with involvement of multiple components within the tumor microenvironment (reviewed in Dovizio et $\mathrm{a}^{19}$ and Menter et $\mathrm{al}^{20}$ ). Thrombocytosis (PLT $\geq 400 \times 10^{9} / \mathrm{L}$ ) has been generally recognized as an independent risk factor for reduced survival. ${ }^{21}$ On the other hand, given the wide range of normal values for PLT, attempts were made for its stratification in order to define high-risk groups of patients. For example, the PLT cutoff point determined, using a ROC curve, for 425 patients with esophageal squamous cell carcinoma was $205 \times 10^{9} / \mathrm{L} .{ }^{5}$ Thus, patients with PLT $\leq 205 \times 10^{9} / \mathrm{L}$ had a significantly better 5 -year survival rate than patients with a PLT $>205 \times 10^{9} / \mathrm{L}$. In this study, the PLT cutoff point could not be determined; so $400 \times 10^{9} / \mathrm{L}$ was used for prognostic analysis. Although no significant association of PLT was found with prognosis, its levels in general were highly correlated with the NLR and $\mathrm{RDW}$ ones. Larger prospective studies are required to determine the prognostic relevance of PLT.

One major finding is a significant association of RDW and NLR with outcomes in glioma patients as well as a strong association of these parameters with one another and with tumor grade. However, the role of RDW and NLR as independent prognostic factors was not revealed in this study. A possible explanation for this finding is that a large number of patients with brain tumors take nonsteroidal antiinflammatory drugs for relieving headache. In this case, the role of inflammation markers such as NLR may be masked. ${ }^{22}$ Further studies with larger cohorts of patients are therefore needed to achieve appropriate statistical results.

The key point of survival analysis is cutoff optimization. Thus, the present study achieved better results using the optimized cutoff value for RDW (13.95), as compared to the recommended value of 14.5. Similarly, optimization of the cutoff for the age (45 years) has led to improved results in comparison with the commonly used cutoff of 65 years (data not shown).

\section{Limitations}

First, the retrospective nature of this study and, therefore, lack of control over data collection may be a source of potential bias. Certain variables such as tumor residual volume and extent of resection were not included in the prognostic analysis as the data were not available for all patients. These factors appear to contribute to prognosis estimation. ${ }^{23}$ Another possible confounding factor is the heterogeneity in patient population and treatment. Finally, due to lack of data, this study could not compare the prognostic relevance of NLR and RDW to that of the Glasgow Prognostic Score, an inflammation-based prognostic score which measures circulating C-reactive protein and albumin (reviewed in McMillan ${ }^{24}$ ). Such analyses should be performed in future studies.

\section{Conclusion}

This study demonstrated the utility of preoperative NLR and RDW values in predicting prognosis for glioma patients. There are several important implications of these findings. First, due to NLR and RDW's wide accessibility and costefficiency, they may help in identifying patients at a high risk of poor prognosis and consequently in selecting an appropriate treatment strategy and follow-up of patients. Second, a large body of evidence highlights the importance of developing anti-inflammatory strategies for cancer treatment. In this context, two large studies should be mentioned; the authors have shown that aspirin taken for several years (at doses of at least $75 \mathrm{mg}$ daily) reduced long-term incidence and mortality due to colorectal and other types of cancer. ${ }^{25,26}$ Finally, focused efforts are required to understand the mechanisms underlying the alterations of NLR and RDW in carcinogenesis and, overall, in chronic inflammatory conditions.

\section{Acknowledgment}

This study was supported by Ministry of Education and Science of the Republic of Kazakhstan (grant number 5377/GF4).

\section{Disclosure}

The authors report no conflicts of interest in this work.

\section{References}

1. Trinchieri G. Cancer and inflammation: an old intuition with rapidly evolving new concepts. Annu Rev Immunol. 2012;30:677-706.

2. Hasselbalch HC. Chronic inflammation as a promotor of mutagenesis in essential thrombocythemia, polycythemia vera and myelofibrosis. A human inflammation model for cancer development? Leuk Res. 2013; $37: 214-220$. 
3. Templeton AJ, McNamara MG, Šeruga B, et al. Prognostic role of neutrophil-to-lymphocyte ratio in solid tumours: a systematic review and meta-analysis. J Natl Cancer Inst. 2014;106(6): dju124.

4. Wan S, Lai Y, Myers RE, et al. Preoperative platelet count associates with survival and distant metastasis in surgically resected colorectal cancer patients. J Gastrointest Cancer. 2013;44(3):293-304.

5. Feng JF, Huang Y, Lu WS, Chen QX. Preoperative platelet count in esophageal squamous cell carcinoma: is it a prognostic factor? Langenbecks Arch Surg. 2013;398:1115-1122.

6. Ishizuka M, Nagata H, Takagi K, et al. Combination of platelet count and neutrophil to lymphocyte ratio is a useful predictor of postoperative survival in patients with colorectal cancer. BJC. 2013;109:401-407.

7. Felker GM, Allen LA, Pocock SJ, et al. Red cell distribution width as a novel prognostic marker in heart failure. JACC. 2007;50(1):40-47.

8. Lee JH, Chung HJ, Kim K, et al. Red cell distribution width as a prognostic marker in patients with community-acquired pneumonia. Am J Emerg Med. 2013;31:72-79.

9. Salvagno GL, Sanchis-Gomar F, Picanza A, Lippi G. Red blood cell distribution width: A simple parameter with multiple clinical applications. Crit Rev Clin Lab Sci. 2015;52(2):86-105.

10. Koma Y, Onishi A, Matsuoka H, et al. Increased red blood cell distribution width associates with cancer stage and prognosis in patients with lung cancer. PLoS One. 2013;8(11):e80240.

11. Warwick R, Mediratta N, Shackcloth M, et al. Preoperative red cell distribution width in patients undergoing pulmonary resections for non-small-cell lung cancer. Eur J Cardiothorac Surg. 2014;45(1): 108-113.

12. Bambury RM, Teo MY, Power DG, et al. The association of pretreatment neutrophil to lymphocyte ratio with overall survival in patients with glioblastoma multiforme. J Neurooncol. 2013;114:149-154.

13. Brockmann MA, Giese A, Mueller K, et al. Preoperative thrombocytosis predicts poor survival in patients with glioblastoma. Neuro Oncol. 2007;9:335-342.

14. Shimada H, Takiguchi N, Kainuma O, et al. High preoperative neutrophil-lymphocyte ratio predicts poor survival in patients with gastric cancer. Gastric Cancer. 2010;13:170-176.

15. Sionov RV, Fridlender ZG, Granot Z. The multifaceted roles neutrophils play in the tumour microenvironment. Cancer Microenviron. 2015;8(3):125-128.
16. Förhécz Z, Gombos T, Borgulya G, Pozsonyi Z, Prohászka Z, Jánoskuti L. Red cell distribution width in heart failure: prediction of clinical events and relationship with markers of ineffective erythropoiesis, inflammation, renal function, and nutritional state. Am Heart J. 2009;158(4):659-666.

17. Lappe JM, Horne BD, Shah SH, et al. Red cell distribution width, $\mathrm{C}$-reactive protein, the complete blood count, and mortality in patients with coronary disease and a normal comparison population. Clin Chim Acta. 2011;412:2094-2099.

18. Patel KV, Semba RD, Ferrucci L, et al. Red cell distribution width and mortality in older adults: a meta-analysis. J Gerontol A Biol Sci Med Sci. 2010;65(3):258-265.

19. Dovizio M, Alberti S, Guillem-Llobat P, et al. Role of platelets in inflammation and cancer: novel therapeutic strategies. Basic Clin Pharmacol Toxicol. 2014;114:118-127.

20. Menter DG, Tucker SC, Kopetz S, Sood AK, Crissman JD, Honn KV. Platelets and cancer: a casual or causal relationship: revisited. Cancer Metastasis Rev. 2014;33(1):231-269.

21. Ma X, Wang Y, Sheng H, et al. Prognostic significance of thrombocytosis, platelet parameters and aggregation rates in epithelial ovarian cancer. J Obstet Gynaecol Res. 2014;40(1):178-183.

22. Imtiaz F, Shafique K, Mirza1 SS, Ayoob Z, Vart P, Rao S. Neutrophil lymphocyte ratio as a measure of systemic inflammation in prevalent chronic diseases in Asian population. Int Arch Med. 2012;5:2-6.

23. Ewelt C, Goeppert M, Rapp M, et al. Glioblastoma multiforme of the elderly: the prognostic effect of resection on survival. J Neurooncol. 2011;103:611-618.

24. McMillan DC. The systemic inflammation-based Glasgow Prognostic Score: a decade of experience in patients with cancer. Cancer Treat Rev. 2013;39(5):534-540.

25. Rothwell PM, Wilson M, Elwin CE, et al. Long-term effect of aspirin on colorectal cancer incidence and mortality: 20-year follow-up of five randomized trials. Lancet. 2010;376:1741-1750.

26. Rothwell PM, Fowkes FG, Belch JF, Ogawa H, Warlow CP, Meade TW. Effect of daily aspirin on long-term risk of death due to cancer: analysis of individual patient data from randomized trials. Lancet. 2011; $377: 31-41$.
OncoTargets and Therapy

\section{Publish your work in this journal}

OncoTargets and Therapy is an international, peer-reviewed, open access journal focusing on the pathological basis of all cancers, potential targets for therapy and treatment protocols employed to improve the management of cancer patients. The journal also focuses on the impact of management programs and new therapeutic agents and protocols on

\section{Dovepress}

patient perspectives such as quality of life, adherence and satisfaction. The manuscript management system is completely online and includes a very quick and fair peer-review system, which is all easy to use. Visit http://www.dovepress.com/testimonials.php to read real quotes from published authors. 